\title{
EL SECTOR COOPERATIVO OLEÍCOLA Y EL USO DE LAS TIC: UN ESTUDIO COMPARATIVO RESPECTO A OTRAS FORMAS JURÍDICAS ${ }^{l}$
}

\author{
POR \\ Domingo FERNÁNDEZ UCLÉS, \\ Enrique BERNAL JURADO, \\ Adoración MOZAS MORAL, \\ Miguel Jesús MEDINA VIRUEL y \\ Encarnación MORAL PAJARES ${ }^{2}$
}

\section{RESUMEN}

El actual entorno globalizado y el aumento generalizado de la difusión y de la utilización de las Tecnologías de la Información y Comunicación (TIC) por parte de las empresas y de los consumidores, hace que el uso de estas tecnologías sea fundamental para que el sector cooperativo oleícola afronte la creciente competencia en el mercado. Centrado en dicho sector, en este trabajo analizamos el grado de utilización del comercio electrónico, tanto a través de los sitios Web como mediante los mercados electrónicos y la utilización que estas empresas realizan de las redes sociales como herramientas facilitadoras de la actividad comercial. Para esto, realizamos un análisis comparativo entre las cooperativas oleícolas andaluzas y el resto de formas jurídicas presentes en el sector.

Palabras clave: Sector del aceite de oliva, cooperativas, comercio electrónico, redes sociales online, mercados electrónicos.

Claves ECONLIT: O13, O33, P13, Q13.

\footnotetext{
${ }^{1}$ Este trabajo de investigación ha sido financiado por el proyecto de excelencia de la Junta de Andalucía: "Estrategias de mejora de la comercialización de los aceites de oliva". Código: AGR-6132.

2 Universidad de Jaén. Direcciones de correo electrónico: f.u.domingo@gmail.com, ebernal@ujaen.es, amozas@ujaen.es, mjmedina@ujaen.es y emoral@ujaen.es.

REVESCO No 120 - Primer Cuatrimestre 2016 - ISSN: 1885-8031 - www.ucm.es/info/revesco

http://dx.doi.org/10.5209/rev_REVE.2016.v120.49700

Fecha de recepción: 14/02/2015

Fecha de aceptación: 25/06/2015
} 


\title{
THE SECTOR OF OLIVE-OIL COOPERATIVES AND THE USE OF ICT: A COMPARATIVE STUDY REGARDING OTHER LEGAL FORMS
}

\begin{abstract}
The current global environment and the general increase in the spread and use of Information Technology and Communication (ICT) by companies and consumers, make the use of these technologies as essential to confront the growing competition in the market. Focused on this sector, in this research we analyze the use of electronic commerce, as through websites as through electronic markets, and the use of social networking tools as enablers of business. For this aim, we conducted a comparative analysis between the Andalusian olive oil cooperatives and other legal forms which are present in the sector.
\end{abstract}

Keywords: Olive oil sector, Cooperatives, Electronic commerce, Online social networking, electronic markets.

\section{INTRODUCCIÓN}

El desarrollo tecnológico ha calado en todos los sectores productivos, entre ellos el oleícola (Mozas, Bernal y Murgado, 2007), mejorando los procesos internos de las sociedades cooperativas que de él participan. La difusión e implantación de las Tecnologías de Información y Comunicación (TIC) en el tejido empresarial, ha creado un escenario favorable para el comercio electrónico ${ }^{3}$ que está desplazando lentamente la utilización del canal de compra-venta tradicional. Esta incipiente modalidad de comercio ha perfeccionado y agilizado la gestión de ventas dentro de las empresas, dotándolas de nuevas capacidades para que puedan hacer frente a la influencia de la globalización, que tanto ha afectado al sector del aceite de oliva (Mili y Rodríguez-Zúñiga, 2001).

$\mathrm{Al}$ respecto, Carr (2004) sostiene que la inversión en estas tecnologías no es suficiente, si no se realiza un uso adecuado de ellas. Por su parte, Apigian, Ragu-Nathan, Ragu-Nathan y Kunnathur (2005) consideran que muchas empresas introducen TIC en su organización sin una comprensión clara de cómo el uso de éstas puede hacerlas más competitivas en el mercado.

\footnotetext{
${ }^{3}$ Turban, Lee, King, Liang y Turban (2009) definen el comercio electrónico como el proceso de compra, venta, transferencia de bienes y servicios y/o información a través de Internet.
} 
Una de las potencialidades del uso de las TIC para las empresas es el mejor acceso al comercio exterior (Gómez y Aleixandre, 2014; Medina, Mozas, Bernal y Moral, 2014), dado que el comercio electrónico ha reducido los costes de transacción y ha facilitado el contacto entre usuarios de cualquier parte del mundo al eliminar las barreras geográficas que existían en el comercio tradicional (Liberos, Somalo, Gil, Gil, García y Merino, 2011). De esta manera, el uso de las TIC es especialmente importante para que las PYMEs puedan aprovechar plenamente las oportunidades que les ofrece el mercado exterior (Sadowski, Mailand y Van Dongen, 2002). Este tipo de empresas, por sus limitados recursos humanos y financieros, ven limitado su acceso a los mercados internacionales. Ante esta situación, el uso de las TIC resulta fundamental para minimizar los efectos de estos condicionantes estructurales, facilitándoles la internacionalización (Stansfields y Grant, 2003; Rasheed, 2005).

En este contexto se encuadra el trabajo de investigación que se presenta. En él se realiza un análisis sobre el uso de las TIC en las sociedades cooperativas oleícolas de la comunidad andaluza, principalmente de su participación en el comercio electrónico (tiendas electrónicas en el sitio Web y mercados electrónicos) y la utilización de las redes sociales como herramientas de ayuda a la comercialización de sus productos. Por tanto, el principal objetivo es comprobar si las sociedades cooperativas oleícolas andaluzas están realizando un uso adecuado de estas herramientas que pueda ayudar a su avance comercial.

Para alcanzar dicho objeto y poder evaluar el uso de las TIC en las sociedades cooperativas oleícolas andaluzas, hemos realizado un análisis comparativo, entre los datos obtenidos en las sociedades cooperativas con el resto de empresas del mismo sector y ámbito de actuación, pero de distinta forma jurídica.

El método empleado es de carácter descriptivo, obteniendo los datos a través de encuestas telefónicas dirigidas a los responsables de las empresas oleícolas de Andalucía con sitio Web. En el siguiente apartado se especifica la población objeto de estudio y la muestra seleccionada.

La secuencia de la investigación es la siguiente: en este apartado hemos delimitado nuestro ámbito de investigación, definido el objetivo perseguido y avanzado la metodología y población del estudio. En el siguiente definimos, de manera más exhaustiva, la metodología seguida en esta investigación, destacando el estudio empírico realizado. Posteriormente, en 
sucesivos apartados, analizamos el uso que, las sociedades cooperativas oleícolas andaluzas están realizando en la actualidad del comercio electrónico, través de sus sitios Web y de los mercados electrónicos, y de las redes sociales, llevando a cabo una comparativa con el uso que realizan las empresas de otras formas jurídicas. El trabajo termina con las conclusiones y las referencias bibliográficas.

\section{METODOLOGÍA}

La metodología seguida para la realización de esta investigación ha sido de tipo descriptivo, analizando los datos sobre el uso de las TIC con fines comerciales de las empresas oleícolas andaluzas, diferenciando entre sociedades cooperativas y no cooperativas. Para esto hemos partido de las sociedades oleícolas andaluzas dedicadas a la comercialización de aceite de oliva, con sitio Web propio. Esta población fue obtenida de la siguiente forma: se partió del directorio de empresas andaluzas comercializadoras de productos oleícolas, censo elaborado y disponible en la Web de la Consejería de Agricultura, Pesca y Medio Ambiente de la Junta de Andalucía. Posteriormente, identificamos aquéllas empresas que disponían de sitio Web, a partir de los principales buscadores de Internet. Como vemos en la tabla 1, del total de empresas del censo (1.029), 440 disponían de sitio Web propio. De éstas, 213 eran sociedades cooperativas y 227 otro tipo de sociedades. Estas 440 empresas son las que constituyen la nuestra población objeto de estudio.

Tabla 1. Empresas andaluzas productoras de aceite de oliva, según su forma jurídica y su tenencia de página $W e b$

\begin{tabular}{|l|c|c|c|}
\hline & S.C.A. & Otras F.J. & TOTAL \\
\hline Productoras de aceite de oliva & 408 & 621 & 1.029 \\
\hline Empresas con página $\mathrm{Web}$ & 213 & 227 & 440 \\
\hline
\end{tabular}

Fuente: Elaboración propia

A estas 440 sociedades oleícolas andaluzas con sitio Web propio se les realizó una encuesta telefónica para obtener los datos necesarios para nuestra investigación, obteniendo respuesta de 214 de ellas (125 sociedades cooperativas y 89 sociedades con otra forma jurídica), teniendo este estudio un error muestral del 4,90 por 100 (ver tabla 2).

Tabla 2. Ficha técnica del estudio empírico

\begin{tabular}{|r|l|}
\hline POBLACIÓN & \\
\hline Unidades de muestreo: & Sociedades Oleícolas con página Web. \\
\hline Población total: & 440 Sociedades \\
\hline
\end{tabular}




\begin{tabular}{|r|l|}
\hline Elementos de muestreo: & $\begin{array}{l}\text { Máximos responsables de la administración de las } \\
\text { sociedades o aquellas personas a las que nos dirigía el } \\
\text { anterior (miembros del Consejo Rector o } \\
\text { Responsables de la Oficina). }\end{array}$ \\
\hline Alcance: & Comunidad Autónoma de Andalucía. \\
\hline Tiempo: & 1 de diciembre de 2012 al 5 de abril de 2013. \\
\hline TUESTREO: & Aleatorio Simple. \\
\hline Tamaño muestral: & 214 sociedades \\
\hline Encuestas válidas: & 214 sociedades \\
\hline Error muestral & 4,90 por 100, para $\mathrm{p}=\mathrm{q}=0,5$ y un nivel de confianza \\
aproximado: & del 95,5 por 100 y factor de corrección. \\
\hline
\end{tabular}

Fuente: Elaboración propia

Una vez descritos los objetivos y la metodología empleada en este trabajo de investigación, pasaremos al siguiente bloque, en el que se exponen y comentan los resultados obtenidos.

\section{EL COMERCIO ELECTRÓNICO: ANÁlISIS MEDIANTE EL USO DE LOS SITIOS WEB}

El comercio electrónico ofrece grandes posibilidades comerciales y beneficios, tanto para las empresas, como para sus clientes. Basándonos en investigaciones sobre el comercio electrónico, como las de Ponce y Escanciano (1997), Casares (1998), Jiménez, Del Águila y Padilla (2000), Sellers y Azorín (2001), Zuang y Lederer (2003), Mozas (2004) o INTECO (2010), podemos establecer, en la tabla 3, una serie de ventajas que el comercio electrónico ofrece a sus usuarios.

Tabla 3. Ventajas del comercio electrónico

\begin{tabular}{|l|l|}
\hline \multicolumn{1}{|c|}{ Ventajas para las empresas } & \multicolumn{1}{|c|}{ Ventajas para los consumidores } \\
\hline - Presencia global. & - Elección global. \\
- Mejora de competitividad. & - Personalización de bienes. \\
- Reducción de costes de compra y transacción. & - Reducción de precios. \\
-Nuevas oportunidades de negocio. & - Rápida respuesta a las necesidades. \\
- Reducción o eliminación de intermediarios. & - Facilidad y comodidad de compra. \\
- Mejora de la comunicación. & - Acceso a nuevos productos. \\
- Feedback con los usuarios. & - Mejora de la calidad de los \\
- Servicios personalizados con clientes y & servicios. \\
proveedores. & - Atención personalizada. \\
- Obtención de economías de alcance y escala. & \\
\hline
\end{tabular}

Fuente: Elaboración propia 
Además de estas ventajas, el comercio electrónico ofrece otras ventajas comunes tanto para las empresas como para los consumidores, como son la rapidez en la compra-venta y el ser un mercado abierto las 24 horas del día y los 365 días del año.

Por otra parte, las empresas deben ser conscientes de que el comercio electrónico no está exento de inconvenientes. Entre estos, caben destacar la falta de contacto físico entre los contratantes, lo que conlleva la falta de confianza y seguridad, problemas o dificultades con la distribución, la realización de pagos, la gestión de reclamaciones o las devoluciones, entre otros (INTECO, 2010).

Una vez que las empresas son conocedoras de las ventajas e inconvenientes del uso del comercio electrónico y deciden participar en él, pueden hacerlo, fundamentalmente, de dos maneras distintas: a través de las tiendas electrónicas situadas en sus sitios Web (comercio electrónico directo) o mediante los mercados electrónicos (comercio electrónico indirecto). En este apartado, comenzamos analizando las transacciones realizadas por las empresas haciendo uso de las tiendas electrónicas ${ }^{4}$, diferenciando entre compras y ventas online.

\subsection{Compras en sitios Web}

En esta sección se pregunta a las empresas si efectúan compras a través de tiendas electrónicas. Aquéllas con respuesta positiva se las cataloga según el importe de dichas adquisiciones y el origen de la compra. Por su parte, las que dan una respuesta negativa nos indican los principales motivos por los que no desempeñan tal actividad.

Según el informe ePYME de Fundetec (2012), el sector agroalimentario en España tiene una incorporación y utilización de TIC superior a la media nacional. Según este informe, un 44,50 por 100 de las empresas del sector agroalimentario realizan compras en tiendas virtuales, frente al 23,30 por 100 del total de la economía española.

Los resultados de nuestro estudio muestran lo siguiente: en la tabla 4 se recoge el número de empresas que efectúan compras a través de la Web, diferenciando entre sociedades de carácter cooperativo y demás formas jurídicas. En ella se puede observar como más de la mitad de las empresas estudiadas no acuden a las plataformas Web para abastecerse (un 57,94 por 100 de los casos). En este sentido, se observa un uso ligeramente superior en las

\footnotetext{
${ }^{4}$ Las tiendas electrónicas son espacios insertos en los sitios Web de las empresas que ofrecen servicios de promoción y marketing de productos, así como la posibilidad de realizar pedidos y pagar o cobrar el importe de las transacciones (Jiménez et al., 2000).
}

REVESCO No 120 - Primer Cuatrimestre 2016 - ISSN: 1885-8031 - www.ucm.es/info/revesco 
sociedades cooperativas, con una diferencia de tan solo 0,83 puntos porcentuales con el resto de organizaciones, siendo en ambos casos ligeramente inferior al total del sector agroalimentario español. Sin embargo, estos datos no son tan negativos si los comparamos con estudios anteriores (Mozas y Bernal, 2008; Vázquez, 2010), en los que se muestra niveles ínfimos de compra online. Además, en estudios similares, como el de Montegut, Cristóbal y Gómez (2013), se muestra cómo el principal uso de Internet está relacionado con la comunicación (vía email), la información y las transacciones financieras en lugar de las transacciones comerciales.

Tabla 4. Empresas que realizan compras a través de sitios Web

\begin{tabular}{|l|c|c|c|c|c|c|}
\hline \multirow{2}{*}{ Respuesta } & \multicolumn{2}{|c|}{ S.C.A } & \multicolumn{2}{c|}{ Otras F.J. } & \multicolumn{2}{c|}{ TOTAL } \\
\cline { 2 - 7 } & $\mathbf{N}^{\mathbf{0}}$ & $\mathbf{\%}$ & $\mathbf{N}^{\mathbf{0}}$ & $\mathbf{\%}$ & $\mathbf{N}^{\mathbf{0}}$ & $\boldsymbol{\%}$ \\
\hline Sí & 53 & 42,40 & 37 & 41,57 & $\mathbf{9 0}$ & $\mathbf{4 2 , 0 6}$ \\
\hline No & 72 & 57,60 & 52 & 58,43 & $\mathbf{1 2 4}$ & $\mathbf{5 7 , 9 4}$ \\
\hline TOTAL & $\mathbf{1 2 5}$ & $\mathbf{1 0 0 , 0 0}$ & $\mathbf{8 9}$ & $\mathbf{1 0 0 , 0 0}$ & $\mathbf{2 1 4}$ & $\mathbf{1 0 0 , 0 0}$ \\
\hline
\end{tabular}

Fuente: Elaboración propia

Realizando un análisis más pormenorizado, podemos clasificar las empresas atendiendo al porcentaje que el importe de las compras efectuadas a través de sitios Web representa respecto a sus compras totales. Como vemos en la tabla 5, el grueso de las sociedades analizadas hace un uso muy débil del comercio electrónico para comprar, ya que el 76,67 por 100 de ellas realizan compras online por un importe que no supera el 10 por 100 del total de sus compras. Haciendo distinción según la forma jurídica de la organización, vemos cómo, en los intervalos con menor porcentaje de compra a través de sitios Web, las sociedades cooperativas tienen mayor representación respecto de las que no lo son, mientras que, en los intervalos con mayor porcentaje de compra online se invierten los resultados.

Tabla 5. Porcentaje del importe total de compras realizadas a través de sitios Web

\begin{tabular}{|l|c|c|c|c|c|c|}
\hline \multirow{2}{*}{$\begin{array}{l}\text { Porcentaje de compras a través } \\
\text { del comercio electrónico }\end{array}$} & \multicolumn{2}{|c|}{ S.C.A } & \multicolumn{2}{c|}{ Otras F.J. } & \multicolumn{2}{c|}{ TOTAL } \\
\cline { 2 - 7 } & $\mathbf{N}^{\mathbf{0}}$ & $\mathbf{\%}$ & $\mathbf{N}^{\mathbf{0}}$ & $\mathbf{\%}$ & $\mathbf{N}^{\mathbf{0}}$ & $\mathbf{\%}$ \\
\hline Menos de 5 & 24 & 45,28 & 14 & 37,84 & $\mathbf{3 8}$ & $\mathbf{4 2 , 2 2}$ \\
\hline $5-10$ & 19 & 35,85 & 12 & 32,43 & $\mathbf{3 1}$ & $\mathbf{3 4 , 4 5}$ \\
\hline $11-15$ & 2 & 3,77 & 1 & 2,70 & $\mathbf{3}$ & $\mathbf{3 , 3 3}$ \\
\hline $16-20$ & 1 & 1,89 & 3 & 8,11 & $\mathbf{4}$ & $\mathbf{4 , 4 5}$ \\
\hline $21-25$ & 0 & 0,00 & 1 & 2,70 & $\mathbf{1}$ & $\mathbf{1 , 1 1}$ \\
\hline Más de 25 & 6 & 11,32 & 4 & 10,81 & $\mathbf{1 0}$ & $\mathbf{1 1 , 1 1}$ \\
\hline Ns/Nc & 1 & 1,89 & 2 & 5,41 & $\mathbf{3}$ & $\mathbf{3 , 3 3}$ \\
\hline TOTAL & $\mathbf{5 3}$ & $\mathbf{1 0 0 , 0 0}$ & $\mathbf{3 7}$ & $\mathbf{1 0 0 , 0 0}$ & $\mathbf{9 0}$ & $\mathbf{1 0 0 , 0 0}$ \\
\hline
\end{tabular}

Fuente: Elaboración propia 
Continuando con el análisis, la tabla 6 cataloga las empresas estudiadas atendiendo a la procedencia de las adquisiciones realizadas a través de sitios Web. En esta tabla podemos ver cómo más del 90 por 100 de las compras a través de websites tienen como origen el mercado nacional y solamente un 8,81 por 100 se realizan en sitios Web de procedencia extranjera, especialmente de Europa. Ese porcentaje se vuelve más dispar si solo nos centramos en sociedades de tipo cooperativo, en el que las compras en sitios Web foráneas no alcanzan ni tan siquiera el 5 por 100 del total.

Tabla 6. Origen de las compras realizadas por las empresas a través de sitios Web (porcentaje)

\begin{tabular}{|l|c|c|c|}
\hline Área geográfica & S.C.A. & Otras F.J. & TOTAL \\
\hline España & 93,36 & 88,08 & $\mathbf{9 1 , 1 9}$ \\
\hline Resto de países de la UE & 3,72 & 4,72 & $\mathbf{4 , 1 3}$ \\
\hline Terceros países & 1,04 & 4,49 & $\mathbf{2 , 4 6}$ \\
\hline Ns/Nc & 1,88 & 2,71 & $\mathbf{2 , 2 2}$ \\
\hline TOTAL & $\mathbf{1 0 0 , 0 0}$ & $\mathbf{1 0 0 , 0 0}$ & $\mathbf{1 0 0 , 0 0}$ \\
\hline
\end{tabular}

Fuente: Elaboración propia

Por último, dentro de este apartado referente a compras a través de sitios Web, la tabla 7 recoge los principales motivos que aportan las empresas entrevistadas para abastecerse por medio de esta modalidad de comercio. Como podemos ver, estas justificaciones se mantienen independientemente de la forma jurídica de la empresa, ya sea de carácter cooperativo o no.

Tabla 7. Razones por las que las empresas no realizan compras a través de Internet

\begin{tabular}{|l|c|c|c|c|c|c|}
\hline \multirow{2}{*}{ Razones } & \multicolumn{2}{|c|}{ S.C.A } & \multicolumn{2}{c|}{ Otras F.J. } & \multicolumn{2}{c|}{ TOTAL } \\
\cline { 2 - 8 } & $\mathbf{N}^{\mathbf{0}}$ & $\mathbf{\%}$ & $\mathbf{N}^{\mathbf{o}}$ & $\mathbf{\%}$ & $\mathbf{N}^{\mathbf{0}}$ & $\mathbf{\%}$ \\
\hline La empresa no lo necesita & 48 & 66,67 & 29 & 55,77 & $\mathbf{7 7}$ & $\mathbf{6 2 , 1 0}$ \\
\hline No me lo he planteado & 16 & 22,22 & 15 & 28,85 & $\mathbf{3 1}$ & $\mathbf{2 5 , 0 0}$ \\
\hline $\begin{array}{l}\text { Los productos no son adecuados para } \\
\text { comprarlos por Internet }\end{array}$ & & & & & & \\
\hline Resulta muy costoso & 16 & 22,22 & 12 & 23,08 & $\mathbf{2 8}$ & $\mathbf{2 2 , 5 8}$ \\
\hline Desconfianza & 1 & 1,39 & 6 & 11,54 & $\mathbf{7}$ & $\mathbf{5 , 6 5}$ \\
\hline Los proveedores no tienen la aplicación & 3 & 4,17 & 1 & 1,92 & $\mathbf{4}$ & $\mathbf{3 , 2 3}$ \\
\hline Ns/Nc & 1 & 1,39 & 1 & 1,92 & $\mathbf{2}$ & $\mathbf{1 , 6 1}$ \\
\hline
\end{tabular}

Fuente: Elaboración propia

\subsection{Ventas en sitios Web}

En este apartado vamos a analizar las ventas efectuadas a través de los propios sitios $W e b$ de las empresas, siguiendo el mismo procedimiento que en el apartado anterior. 
Según el informe ePYME de Fundetec (2012), un 24,40 por 100 de las empresas del sector agroalimentario realizan ventas en tiendas virtuales, siendo aún inferior el porcentaje en el total de la economía española (un 12,20 por 100).

Los resultados de nuestro estudio, mostrados en la tabla 8 , ponen de manifiesto un mayor uso de las tiendas electrónicas para realizar ventas online en el sector oleícola ecológico español. Sin embargo, al igual que sucedía con las compras realizadas a través de sitios Web, más de la mitad de las empresas estudiadas no utilizan su sitio Web como medio de venta (52,34 por 100). De igual forma, no existen diferencias significativas discriminando por las sociedades cooperativas, cuyo porcentaje es similar a la media aunque de índole negativa. En cualquier caso, en general la situación ha evolucionado positivamente si atendemos a los resultados de estudios anteriores, como los de Mozas y Bernal (2008), según los cuales hace aproximadamente una década tan solo un 17 por 100 de las empresas oleícolas jiennenses entrevistadas disponían de una Web de carácter transaccional, que permitiera vender sus productos.

Tabla 8. Empresas que realizan ventas a través de sus sitios Web

\begin{tabular}{|l|c|c|c|c|c|c|}
\hline \multirow{2}{*}{ Respuesta } & \multicolumn{2}{|c|}{ S.C.A } & \multicolumn{2}{c|}{ Otras F.J. } & \multicolumn{2}{c|}{ TOTAL } \\
\cline { 2 - 7 } & $\mathbf{N}^{\mathbf{0}}$ & $\mathbf{\%}$ & $\mathbf{N}^{\mathbf{0}}$ & $\boldsymbol{\%}$ & $\mathbf{N}^{\mathbf{0}}$ & $\boldsymbol{\%}$ \\
\hline Sí & 59 & 47,20 & 43 & 48,31 & $\mathbf{1 0 2}$ & $\mathbf{4 7 , 6 6}$ \\
\hline No & 66 & 52,80 & 46 & 51,69 & $\mathbf{1 1 2}$ & $\mathbf{5 2 , 3 4}$ \\
\hline TOTAL & $\mathbf{1 2 5}$ & $\mathbf{1 0 0 , 0 0}$ & $\mathbf{8 9}$ & $\mathbf{1 0 0 , 0 0}$ & $\mathbf{2 1 4}$ & $\mathbf{1 0 0 , 0 0}$ \\
\hline
\end{tabular}

Fuente: Elaboración propia

Encasillando estas empresas según el volumen de ventas realizadas a través de sus plataformas Web (tabla 9) el comercio tradicional es el principal canal de venta de estas empresas. La mayoría de las organizaciones que comercializa a través de su sitio Web factura un importe inferior al 10 por 100 del total de sus ventas, encuadrándose el 41,17 por 100 de las mismas en el intervalo de ventas por comercio electrónico que es inferior al 5 por 100, lo que denota el poco aprovechamiento de este medio de compra-venta online. También se muestra cómo las sociedades cooperativas tienen mayor presencia en los intervalos con menor porcentaje de venta online, mientras que un 13,95 por 100 de las empresas no cooperativas efectúan ventas online por un importe que representa más de una cuarta parte del total de su facturación. 
Tabla 9. Porcentaje del importe total de ventas realizadas a través de sus sitios Web

\begin{tabular}{|l|c|c|c|c|c|c|}
\hline \multirow{2}{*}{$\begin{array}{l}\text { Porcentaje de ventas a través } \\
\text { del comercio electrónico }\end{array}$} & \multicolumn{2}{|c|}{ S.C.A } & \multicolumn{2}{c|}{ Otras F.J. } & \multicolumn{2}{c|}{ TOTAL } \\
\cline { 2 - 7 } & $\mathbf{N}^{\mathbf{0}}$ & $\mathbf{\%}$ & $\mathbf{N}^{\mathbf{0}}$ & $\mathbf{\%}$ & $\mathbf{N}^{\mathbf{0}}$ & $\mathbf{\%}$ \\
\hline Menos de 5 & 26 & 44,07 & 16 & 37,21 & $\mathbf{4 2}$ & $\mathbf{4 1 , 1 7}$ \\
\hline $5-10$ & 18 & 30,51 & 11 & 25,58 & $\mathbf{2 9}$ & $\mathbf{2 8 , 4 3}$ \\
\hline $11-15$ & 2 & 3,39 & 7 & 16,28 & $\mathbf{9}$ & $\mathbf{8 , 8 3}$ \\
\hline $16-20$ & 5 & 8,47 & 3 & 6,98 & $\mathbf{8}$ & $\mathbf{7 , 8 4}$ \\
\hline $21-25$ & 3 & 5,09 & 0 & 0,00 & $\mathbf{3}$ & $\mathbf{2 , 9 4}$ \\
\hline Más de 25 & 3 & 5,09 & 6 & 13,95 & $\mathbf{9}$ & $\mathbf{8 , 8 3}$ \\
\hline Ns/Nc & 2 & 3,39 & 0 & 0,00 & $\mathbf{2}$ & $\mathbf{1 , 9 6}$ \\
\hline TOTAL & $\mathbf{5 9}$ & $\mathbf{1 0 0 , 0 0}$ & $\mathbf{4 3}$ & $\mathbf{1 0 0 , 0 0}$ & $\mathbf{1 0 2}$ & $\mathbf{1 0 0 , 0 0}$ \\
\hline
\end{tabular}

Fuente: Elaboración propia

En la tabla 10 se observa el área geográfica a la que se destinan las ventas efectuadas a través de los sitios Web.

El mercado nacional absorbe casi la totalidad de las ventas que se producen a través del sitio Web de las empresas estudiadas. La exportación solo abarca el 3,31 por 100 de las ventas y se centra casi en su totalidad en la Unión Europea. Atendiendo exclusivamente a las sociedades cooperativas, esta situación se enfatiza y las exportaciones solo representan un 1,90 por 100 del total de ventas. En esta línea, debemos apuntar que diversos autores (Vázquez, 2010; Bruque, Hernández y Vargas, 2010; Moral, Bernal y Alba, 2010) coinciden en resaltar los incalculables beneficios de Internet y de las tiendas electrónicas para las empresas oleícolas españolas (en el acceso a un mercado global, el contacto con numerosos clientes en los mercado internacionales...), a la vez que determinan que las empresas de este sector no realizan un uso estratégico de la Web, sino más bien de carácter estándar y limitado, lo que impide aprovechar los beneficios de Internet al limitar su alcance.

Tabla 10. Destino de las ventas realizadas por las empresas a través de sus sitios Web (porcentaje)

\begin{tabular}{|l|c|c|c|}
\hline Área geográfica & S.C.A. & Otras F.J. & TOTAL \\
\hline España & 96,41 & 94,75 & $\mathbf{9 5 , 7 1}$ \\
\hline Resto de países de la UE & 1,73 & 4,48 & $\mathbf{2 , 8 9}$ \\
\hline Terceros países & 0,17 & 0,77 & $\mathbf{0 , 4 2}$ \\
\hline Ns/Nc & 1,69 & 0,00 & $\mathbf{0 , 9 8}$ \\
\hline TOTAL & $\mathbf{1 0 0 , 0 0}$ & $\mathbf{1 0 0 , 0 0}$ & $\mathbf{1 0 0 , 0 0}$ \\
\hline
\end{tabular}

Fuente: Elaboración propia

Continuando con el análisis de las ventas realizadas a través de los sitios Web, es importante detallar el agente económico al que se destinan. Principalmente, existen tres 
posibles alternativas: rama $\mathrm{B} 2 \mathrm{~B}$ o comercio entre empresas; rama $\mathrm{B} 2 \mathrm{C}$ o comercio dirigido al consumidor final y rama $\mathrm{B} 2 \mathrm{G}$, que comprende las actividades comerciales con las administraciones públicas.

Como se aprecia en la tabla 11, la mayor parte de las ventas online que realizan las empresas a través de su sitio Web se dirigen al consumidor final, en el 83,93 por 100 de los casos. El 12,82 por 100 de las ventas responden al mercado interempresarial y solamente el 1,96 por 100 de las transacciones van a parar a las administraciones públicas. En las sociedades cooperativas la distribución es similar, aunque el mercado dirigido al consumidor final acumula un mayor peso. No obstante, cuando comparamos estos datos con los que nos proporciona el ONTSI (Urueña et al., 2013) a nivel nacional, observamos cómo se invierten las tornas, es decir, las ventas en comercio electrónico B2B adquieren bastante más peso que las $\mathrm{B} 2 \mathrm{C}, 89,50$ por 100 frente al 7,40 por 100, respectivamente, mientras que las de tipo B2G se mantienen en la misma línea, 3,10 por 100.

Tabla 11. Ventas realizadas a través de los sitios Web de las empresas, por tipo de transacción

\begin{tabular}{|l|c|c|c|}
\hline Tipo de transacción & S.C.A. & Otras F.J. & TOTAL \\
\hline B2B & 9,93 & 16,78 & $\mathbf{1 2 , 8 2}$ \\
\hline B2C & 87,75 & 78,69 & $\mathbf{8 3 , 9 3}$ \\
\hline B2G & 0,63 & 2,20 & $\mathbf{1 , 2 9}$ \\
\hline Ns/Nc & 1,69 & 2,33 & $\mathbf{1 , 9 6}$ \\
\hline TOTAL & $\mathbf{1 0 0 , 0 0}$ & $\mathbf{1 0 0 , 0 0}$ & $\mathbf{1 0 0 , 0 0}$ \\
\hline
\end{tabular}

Fuente: Elaboración propia

Para concluir con este apartado se exponen, en la tabla 12, los principales motivos que manifiestan las empresas para no hacer uso de su Web como canal de venta.

En este caso, las razones a las que apuntan los responsables de cada organización para no hacer uso de la venta online varían según se trate de una sociedad cooperativa o no. En las sociedades cooperativas analizadas se percibe insuficiente presión para potenciar el comercio electrónico, al ser los principales motivos señalados la falta de necesidad y el no habérselo planteado. En cambio, el resto de organizaciones manifiestan, con una frecuencia del 47,83 por 100 , que esa actividad se encuentra en proceso. 
Tabla 12. Razones por las que las empresas no realizan ventas a través de Internet

\begin{tabular}{|c|c|c|c|c|c|c|}
\hline \multirow{2}{*}{ Razones } & \multicolumn{2}{|c|}{ S.C.A } & \multicolumn{2}{|c|}{ Otras F.J. } & \multicolumn{2}{|c|}{ TOTAL } \\
\hline & $\mathbf{N}^{\mathbf{o}}$ & $\%$ & $\mathbf{N}^{\mathbf{0}}$ & $\%$ & $\mathbf{N}^{\mathbf{0}}$ & $\%$ \\
\hline No me lo he planteado & 17 & 25,76 & 10 & 21,74 & 27 & 24,11 \\
\hline En proceso & 4 & 6,06 & 22 & 47,83 & 26 & 23,21 \\
\hline La empresa no lo necesita & 17 & 25,76 & 3 & 6,52 & 20 & 17,86 \\
\hline $\begin{array}{l}\text { Lo realiza a través de la cooperativa de } \\
\text { segundo grado }\end{array}$ & 11 & 16,67 & 0 & 0,00 & 11 & $\mathbf{9 , 8 2}$ \\
\hline $\begin{array}{l}\text { Los productos no son adecuados para } \\
\text { comprarlos por Internet }\end{array}$ & 2 & 3,03 & 3 & 6,52 & 5 & 4,46 \\
\hline Dimensión de la empresa & 2 & 3,03 & 0 & 0,00 & 2 & 1,79 \\
\hline Resulta muy costoso & 1 & 1,52 & 1 & 2,17 & 2 & 1,79 \\
\hline Falta de tiempo y personal & 1 & 1,52 & 1 & 2,17 & 2 & 1,79 \\
\hline Los clientes no lo ven adecuado & 1 & 1,52 & 1 & 2,17 & 2 & 1,79 \\
\hline Problemas con los distribuidores & 1 & 1,52 & 1 & 2,17 & 2 & 1,79 \\
\hline La experiencia anterior no tuvo éxito & 0 & 0,00 & 2 & 4,35 & 2 & 1,79 \\
\hline Problemas con la formas de pago & 1 & 1,52 & 0 & 0,00 & $\mathbf{1}$ & $\mathbf{0 , 8 9}$ \\
\hline Desconfianza & 1 & 1,52 & 0 & 0,00 & 1 & $\mathbf{0 , 8 9}$ \\
\hline No están preparados & 1 & 1,52 & 0 & 0,00 & $\mathbf{1}$ & $\mathbf{0 , 8 9}$ \\
\hline $\begin{array}{l}\text { Problemas por la disparidad de precios en } \\
\text { los mercados }\end{array}$ & 0 & 0,00 & 1 & 2,17 & 1 & $\mathbf{0 , 8 9}$ \\
\hline $\mathrm{Ns} / \mathrm{Nc}$ & 2 & 3,03 & 0 & 0,00 & 2 & 1,79 \\
\hline
\end{tabular}

Fuente: Elaboración propia

\section{USOS DE LAS REDES SOCIALES}

Este epígrafe recoge la investigación referente a las empresas que están presentes en redes sociales. En primer lugar, haremos una revisión de la literatura para conocer las aportaciones y consideraciones que estudios anteriores han arrojado sobre el tema. Acto seguido se expondrán los resultados del estudio en el que se determina el uso de estas redes, la importancia que se les concede, su utilidad para la actividad empresarial y las herramientas sociales más utilizadas.

En primer lugar, debemos resaltar la gran difusión y utilización de Internet por parte de la población, que se ha convertido en un gran escaparate en el que los consumidores van a tener acceso a multitud de opciones para realizar sus compras. Es por ello que las empresas deben prestar una especial atención a cómo la organización es percibida por parte del público usuario de Internet, dado que, en la mayoría de los casos, la imagen que proyecte la empresa en Internet, será la que tengan los usuarios de ella. Es decir, las empresas deben cuidar su "reputación online" y un buen mecanismo para ello es hacer uso de las redes sociales, que ayudan a la organización a forjar, crear o modificar su imagen en el entorno virtual (Gallart, 
2010). Este fenómeno es conocido bajo las siglas SMO (Social Media Optimization) e incluye los procesos de marketing destinados a mejorar el posicionamiento de la empresa y su reputación corporativa en las redes sociales, dada la importancia y repercusión de estas comunidades online para la actividad empresarial, comercial y relacional de la organización (Neti, 2011).

Además, las redes sociales se convierten en una poderosa fuente de información para conocer el perfil y las características de los consumidores y así poder establecer una estrategia de marketing adaptada al cliente (Celaya, 2008). Otro de los beneficios de estas herramientas sociales para las organizaciones es la posibilidad de interactuar con los consumidores y obtener una retroalimentación constante e inmediata (O'reilly, 2005). Así, la aparición de las redes sociales debe provocar un cambio radical en la forma en que las empresas dan a conocer sus productos y su organización, proporcionando Internet una alternativa de comunicación de bajo coste (Gunelius, 2011), mejorando la interacción y la confianza con el consumidor (Lai, Tong y Lai, 2011) y las ventas de los productos (Cheng y Xie, 2008 y Wei, Zhang y Sutanto, 2013). Igualmente, Chui, Manyika, Bughin, Dobbs, Roxburgh, Sarrazin, Sands y Westergren (2012) estiman que la plena implementación de las empresas en redes sociales permitiría incrementar la productividad de los trabajadores, en materia de conocimiento, entre un 20 y el 25 por 100 .

En la tabla 13 se indica el número de empresas analizadas que poseen cuentas activas en sitios Web de redes sociales y hacen un uso habitual de ellas. Como vemos, más de la mitad de las organizaciones oleícolas encuestadas no frecuentan las redes sociales, especialmente aquellas que son de rango cooperativo, en el que la cifra se reduce hasta el 40,80 por 100. Con estos datos, este tipo de empresas se situarían por debajo de la media española, si lo comparamos con el estudio de Cink Shaking Business (2013) que determina que un 55,30 por 100 de las PYMEs españolas hace uso de las redes sociales de manera profesional. Esto supone, por tanto, una oportunidad de mercado bastante accesible que no está siendo aprovechada por las empresas de este sector oleícola.

Tabla 13. ¿Su empresa hace uso habitual de las redes sociales? (por forma jurídica)

\begin{tabular}{|l|c|c|c|c|c|c|}
\hline \multirow{2}{*}{ Respuesta } & \multicolumn{2}{|c|}{ S.C.A } & \multicolumn{2}{c|}{ Otras F.J. } & \multicolumn{2}{c|}{ TOTAL } \\
\cline { 2 - 7 } & $\mathbf{N}^{\mathbf{0}}$ & $\mathbf{\%}$ & $\mathbf{N}^{\mathbf{0}}$ & $\mathbf{\%}$ & $\mathbf{N}^{\mathbf{0}}$ & $\boldsymbol{\%}$ \\
\hline Sí & 51 & 40,80 & 44 & 49,44 & $\mathbf{9 5}$ & $\mathbf{4 4 , 3 9}$ \\
\hline No & 74 & 59,10 & 45 & 50,56 & $\mathbf{1 1 9}$ & $\mathbf{5 5 , 6 1}$ \\
\hline TOTAL & $\mathbf{1 2 5}$ & $\mathbf{1 0 0 , 0 0}$ & $\mathbf{8 9}$ & $\mathbf{1 0 0 , 0 0}$ & $\mathbf{2 1 4}$ & $\mathbf{1 0 0 , 0 0}$ \\
\hline
\end{tabular}

Fuente: Elaboración propia 
Cuando se les pregunta a los responsables de las compañías si consideran a las redes sociales instrumentos generadores de valor para la empresa, un 71,58 por 100 consideran que sí (ver tabla 14).

En este caso, tal y como vemos en el siguiente cuadro, las cooperativas oleícolas son entre las empresas encuestadas las más conscientes de la generación de valor que implica el uso de estas herramientas sociales. No obstante, en conjunto, el 28,42 por 100 de las organizaciones las considera anodinas para la actividad empresarial, un porcentaje bastante alto si tenemos en cuenta la cantidad de estudios e investigaciones que proclaman justamente lo contrario.

Tabla 14. ¿Considera que su presencia en las redes sociales aporta valor a su negocio? (por razón social)

\begin{tabular}{|l|c|c|c|c|c|c|}
\hline \multirow{2}{*}{ Respuesta } & \multicolumn{2}{|c|}{ S.C.A } & \multicolumn{2}{c|}{ Otras F.J. } & \multicolumn{2}{c|}{ TOTAL } \\
\cline { 2 - 7 } & $\mathbf{N}^{\mathbf{0}}$ & $\mathbf{\%}$ & $\mathbf{N}^{\mathbf{0}}$ & $\mathbf{\%}$ & $\mathbf{N}^{\mathbf{0}}$ & $\mathbf{\%}$ \\
\hline Sí & 40 & 78,43 & 28 & 63,64 & $\mathbf{6 8}$ & $\mathbf{7 1 , 5 8}$ \\
\hline No & 11 & 21,57 & 16 & 36,36 & $\mathbf{2 7}$ & $\mathbf{2 8 , 4 2}$ \\
\hline TOTAL & $\mathbf{5 1}$ & $\mathbf{1 0 0 , 0 0}$ & $\mathbf{4 4}$ & $\mathbf{1 0 0 , 0 0}$ & $\mathbf{9 5}$ & $\mathbf{1 0 0 , 0 0}$ \\
\hline
\end{tabular}

Fuente: Elaboración propia

Por otro lado, la tabla 15 aúna los principales usos empresariales que las empresas encuestadas hacen de las redes sociales. Los resultados muestran cómo la principal utilidad que se hace de las redes sociales es en materia de marketing y relaciones comerciales con clientes, datos que coinciden con el estudio de eCircle y Mediacom Science (2011) del que se desprende que el 44 por 100 de las organizaciones en Europa utiliza las redes sociales para actividades de marketing y comunicación, en el que España se sitúa como país líder, con un 51 por 100 .

Dadas las potencialidades comerciales que ofrecen estas herramientas, resulta lógico que las empresas utilicen las redes sociales para los fines anteriores. No obstante, este medio ofrece, además, otras aplicaciones de interés comercial, como son la posibilidad de analizar a la competencia y de estudiar las tendencias del mercado (Mehrtens, Cragg y Mills, 2001). 
Tabla 15. ¿Qué uso hace de las redes sociales? (por forma jurídica)

\begin{tabular}{|l|c|c|c|c|c|c|}
\hline \multirow{2}{*}{ Usos de las redes sociales } & \multicolumn{2}{|c|}{ S.C.A } & \multicolumn{2}{c|}{ Otras F.J. } & \multicolumn{2}{c|}{ TOTAL } \\
\cline { 2 - 7 } & $\mathbf{N}^{\mathbf{0}}$ & $\mathbf{\%}$ & $\mathbf{N}^{\mathbf{0}}$ & $\mathbf{\%}$ & $\mathbf{N}^{\mathbf{0}}$ & $\mathbf{\%}$ \\
\hline Marketing de mis productos & 39 & 76,47 & 30 & 68,18 & $\mathbf{6 9}$ & $\mathbf{7 2 , 6 3}$ \\
\hline Comercialización de mis productos & 8 & 15,67 & 4 & 9,09 & $\mathbf{1 2}$ & $\mathbf{1 2 , 6 3}$ \\
\hline Contacto e información a los clientes & 35 & 68,63 & 31 & 70,45 & $\mathbf{6 6}$ & $\mathbf{6 9 , 4 7}$ \\
\hline Contacto con proveedores & 5 & 9,80 & 6 & 13,64 & $\mathbf{1 1}$ & $\mathbf{1 1 , 5 8}$ \\
\hline Análisis de las tendencias del mercado & 7 & 13,73 & 7 & 15,91 & $\mathbf{1 4}$ & $\mathbf{1 4 , 7 4}$ \\
\hline Análisis de competidores & 6 & 11,76 & 7 & 15,91 & $\mathbf{1 3}$ & $\mathbf{1 3 , 6 8}$ \\
\hline Búsqueda de personal & 2 & 3,92 & 0 & 0,00 & $\mathbf{2}$ & $\mathbf{2 , 1 1}$ \\
\hline Informar a los socios & 3 & 5,88 & 0 & 0,00 & $\mathbf{3}$ & $\mathbf{3 , 1 6}$ \\
\hline Ampliar mercado & 1 & 1,96 & 0 & 0,00 & $\mathbf{1}$ & $\mathbf{1 , 0 5}$ \\
\hline Fomentar las relaciones internas & 2 & 3,92 & 0 & 0,00 & $\mathbf{2}$ & $\mathbf{2 , 1 1}$ \\
\hline Simplemente estar presentes & 1 & 1,96 & 6 & 13,64 & $\mathbf{7}$ & $\mathbf{7 , 3 7}$ \\
\hline $\begin{array}{l}\text { Promocionar la cultura del aceite de } \\
\text { oliva }\end{array}$ & 1 & 1,96 & 0 & 0,00 & $\mathbf{1}$ & $\mathbf{1 , 0 5}$ \\
\hline Ns/Nc & 2 & 3,92 & 1 & 2,27 & $\mathbf{3}$ & $\mathbf{3 , 1 6}$ \\
\hline
\end{tabular}

Fuente: Elaboración propia

Continuando con el estudio de las redes sociales en el sector oleícola, es necesario conocer cuáles son las más utilizadas por las empresas.

Los datos mostrados en la tabla 16 son lógicos, dado que Facebook es la red social más extendida en el mundo, con más de 1.190 millones de cuentas (Esteban y Quirós, 2014) y traducida a más de 70 idiomas (Fernández, 2011). Por su parte, Twitter ocupa el cuarto lugar en el ranking de redes sociales generales.

Tabla 16. ¿Cuál es la red social que utiliza más frecuentemente? (por forma jurídica)

\begin{tabular}{|l|c|c|c|c|c|c|}
\hline \multirow{2}{*}{ Red social } & \multicolumn{2}{|c|}{ S.C.A } & \multicolumn{2}{c|}{ Otras F.J. } & \multicolumn{2}{c|}{ TOTAL } \\
\cline { 2 - 7 } & $\mathbf{N}^{\mathbf{0}}$ & $\mathbf{\%}$ & $\mathbf{N}^{\mathbf{0}}$ & $\mathbf{\%}$ & $\mathbf{N}^{\mathbf{0}}$ & $\mathbf{\%}$ \\
\hline Facebook & 36 & 70,59 & 37 & 84,09 & $\mathbf{7 3}$ & $\mathbf{7 6 , 8 5}$ \\
\hline Tuenti & 1 & 1,96 & 0 & 0,00 & $\mathbf{1}$ & $\mathbf{1 , 0 5}$ \\
\hline Twitter & 4 & 7,84 & 2 & 4,55 & $\mathbf{6}$ & $\mathbf{6 , 3 2}$ \\
\hline Linkedin & 1 & 1,96 & 0 & 0,00 & $\mathbf{1}$ & $\mathbf{1 , 0 5}$ \\
\hline Facebook+Twitter & 6 & 11,76 & 5 & 11,36 & $\mathbf{1 1}$ & $\mathbf{1 1 , 5 8}$ \\
\hline Facebook+Twitter+Linkedin & 1 & 1,96 & 0 & 0,00 & $\mathbf{1}$ & $\mathbf{1 , 0 5}$ \\
\hline Facebook+Tuenti & 1 & 1,96 & 0 & 0,00 & $\mathbf{1}$ & $\mathbf{1 , 0 5}$ \\
\hline Ns/Nc & 1 & 1,96 & 0 & 0,00 & $\mathbf{1}$ & $\mathbf{1 , 0 5}$ \\
\hline TOTAL & $\mathbf{5 1}$ & $\mathbf{1 0 0 , 0 0}$ & $\mathbf{4 4}$ & $\mathbf{1 0 0 , 0 0}$ & $\mathbf{9 5}$ & $\mathbf{1 0 0 , 0 0}$ \\
\hline
\end{tabular}

Fuente: Elaboración propia 


\section{CONOCIMIENTO Y UTILIZACIÓN DE LOS MERCADOS ELECTRÓNICOS}

En opinión de Trabold (2002), la aparición de estos intermediarios comerciales en Internet (mercados electrónicos ${ }^{5}$ o eMarketplaces) es resultado del desarrollo del comercio electrónico. Atendiendo a las partes que participan en el mercado electrónico se puede hacer la siguiente clasificación (López, 2004): Mercado electrónico independiente ${ }^{6}$, mercado electrónico orientado a la venta ${ }^{7}$ y mercado electrónico orientado a la compra ${ }^{8}$, aunque hay que decir que cada vez aumenta el número de mercados híbridos que surgen a partir de dichas categorías. Según Liberos et al. (2011) los mercados electrónicos aportan numerosos beneficios a las empresas que concurren en ellos, como se detalla en la tabla 17.

Tabla 17. Beneficios aportados por un Marketplace para compradores y vendedores

\begin{tabular}{|c|c|c|}
\hline Beneficios para compradores & $\begin{array}{l}\text { Fuente } \\
\text { valor }\end{array}$ & Beneficios para vendedores \\
\hline $\begin{array}{l}\text { - Mayores volúmenes negociados. } \\
\text { - Cumplimiento interno de contratos. } \\
\text { - Menores precios. }\end{array}$ & $\begin{array}{l}\text { Agregación de } \\
\text { demanda }\end{array}$ & $\begin{array}{l}\text { - Mayores volúmenes contratados. } \\
\text { - Mayor base de clientes. }\end{array}$ \\
\hline $\begin{array}{l}\text { - Menores costes y tiempos de } \\
\text { búsqueda, negociación } \\
\text { procesamiento. } \\
\text { - Acceso a proveedores sin límite } \\
\text { horario. } \\
\text { - Reducción en inversión } \\
\text { tecnológica. }\end{array}$ & $\begin{array}{lr}\text { Eficiencia de } \\
\text { procesos } & / \\
\text { Outsourcing } & / \\
\text { ASP } & \end{array}$ & $\begin{array}{l}\text { - Menores costes y tiempos de } \\
\text { adquisición de clientes y de } \\
\text { procesamiento. } \\
\text { - Acceso de compradores sin } \\
\text { límite horario. } \\
\text { - Reducción en inversión } \\
\text { tecnológica }\end{array}$ \\
\hline $\begin{array}{l}\text { - Reducir costes de inventario. } \\
\text { - Reducir costes de procesamiento. } \\
\text { - Mayores ingresos. }\end{array}$ & $\begin{array}{ll}\text { Integración } & \text { de } \\
\text { la cadena } & \text { de } \\
\text { suministro } & \end{array}$ & $\begin{array}{l}\text { - Gestión mejorada de inventarios. } \\
\text { - Previsión mejorada de la } \\
\text { demanda. } \\
\text { - Altos costes de cambio para el } \\
\text { comprador. }\end{array}$ \\
\hline $\begin{array}{l}\text { - Benchmarking continuo y } \\
\text { mejorado. } \\
\text { - Menor coste de investigación. } \\
\text { - Respuesta competitiva más rápida. }\end{array}$ & $\begin{array}{ll}\text { Agregación de } \\
\text { contenido } / \\
\text { Comunidad }\end{array}$ & $\begin{array}{l}\text { - Benchmarking continuo y } \\
\text { mejorado. } \\
\text { - Investigación continua, a bajo } \\
\text { coste. } \\
\text { - } \begin{array}{l}\text { Respuesta competitiva más } \\
\text { rápida. }\end{array}\end{array}$ \\
\hline - Transparencia de precios & Eficiencia & - Menores costes de venta. \\
\hline
\end{tabular}

\footnotetext{
5 Sandulli (2011) define los mercados electrónicos como espacios virtuales que median entre oferentes y demandantes y ponen a disposición una serie de herramientas informativas que permiten la realización de transacciones de compra y venta entre los agentes participantes.

${ }^{6}$ Es aquel mercado electrónico gestionado por una persona, física o jurídica, que no forma parte de ninguno de los dos bandos en la operación comercial, lo que no le exime de sacar provecho de tal intercambio.

${ }^{7}$ En esta modalidad de mercado electrónico, la gestión es llevada a cabo por un número determinado de vendedores que cooperan para llevar a cabo la transacción de forma eficiente.

${ }^{8}$ Esta tipología de mercado electrónico es similar a la orientada a la venta pero actúa de forma inversa, es decir, en este caso son los que actúan de compradores los que se encargan de gestionar esta plataforma.
}

REVESCO No 120 - Primer Cuatrimestre 2016 - ISSN: 1885-8031 - www.ucm.es/info/revesco 


\begin{tabular}{|c|c|c|}
\hline $\begin{array}{l}\text { inventarios. } \\
\text { - Coste reducido por compras } \\
\text { esporádicas. } \\
\text { - Coste reducido en exceso de } \\
\text { inventario. } \\
\text { - Acceso más rápido a proveedores. }\end{array}$ & mercado & $\begin{array}{l}\text { - Coste reducido por exceso de } \\
\text { inventario. } \\
\text { - Acceso más rápido a } \\
\text { compradores. }\end{array}$ \\
\hline
\end{tabular}

Fuente: Liberos et al. (2011)

En este apartado vamos a analizar la trascendencia que tienen los mercados electrónicos en el tejido cooperativo oleícola andaluz, para tal propósito se pregunta a los responsables de la organizaciones estudiadas si conocen estas plataformas, si tienen presencia en ellas y en tal caso, qué utilidad les proporciona.

A través de la tabla 18, se puede conocer la proporción de empresas cuyos responsables desconocen qué son y en qué consisten los mercados electrónicos. Así, un 52,34 por 100 de los responsables de las empresas encuestadas desconocen qué es un mercado electrónico, cifra que aumenta hasta el 55,60 por 100 cuando los resultados hacen referencia a las sociedades cooperativas. Estos datos son bastantes negativos, ya que los mercados electrónicos son un canal de actuación muy interesante para futuras estrategias de internacionalización y de marketing, al reducir los costes de intercambio y facilitar la comunicación bilateral, con la que se estrecha la relación con el cliente (López, 2004; Cabal, 2013).

Tabla 18. ¿Conoce los mercados electrónicos como canal de internacionalización?

(por razón social)

\begin{tabular}{|l|c|c|c|c|c|c|}
\hline \multirow{2}{*}{ Respuesta } & \multicolumn{2}{|c|}{ S.C.A } & \multicolumn{2}{c|}{ Otras F.J. } & \multicolumn{2}{c|}{ TOTAL } \\
\cline { 2 - 7 } & $\mathbf{N}^{\mathbf{0}}$ & $\mathbf{\%}$ & $\mathbf{N}^{\mathbf{0}}$ & $\mathbf{\%}$ & $\mathbf{N}^{\mathbf{0}}$ & $\mathbf{\%}$ \\
\hline Sí & 58 & 44,40 & 44 & 49,44 & $\mathbf{1 0 2}$ & $\mathbf{4 7 , 6 6}$ \\
\hline No & 67 & 55,60 & 45 & 50,56 & $\mathbf{1 1 2}$ & $\mathbf{5 2 , 3 4}$ \\
\hline TOTAL & $\mathbf{1 2 5}$ & $\mathbf{1 0 0 , 0 0}$ & $\mathbf{8 9}$ & $\mathbf{1 0 0 , 0 0}$ & $\mathbf{2 1 4}$ & $\mathbf{1 0 0 , 0 0}$ \\
\hline
\end{tabular}

Fuente: Elaboración propia

En esa línea se presenta la tabla 19 en la que se determina el número de empresas que deciden participar o no en los mercados electrónicos, partiendo de la premisa de que los conocen. En esta tabla se puede observar como el 35,29 por 100 de las empresas encuestadas opera en los mercados electrónicos, cifra que decrece hasta el 29,31 por 100 cuando solo tomamos como referencia a las sociedades cooperativas. En este sentido, a pesar de la existencia de más de mil mercados electrónicos a nivel mundial, el porcentaje de empresas que hace uso de estos mercados es todavía muy reducido, esperándose un gran aumento en la 
utilización de estas plataformas por parte tanto de las empresas como del sector público (López, 2004).

Tabla 19. ¿Opera en los mercados electrónicos? (por razón social)

\begin{tabular}{|l|c|c|c|c|c|c|}
\hline \multirow{2}{*}{ Respuesta } & \multicolumn{2}{|c|}{ S.C.A } & \multicolumn{2}{c|}{ Otras F.J. } & \multicolumn{2}{c|}{ TOTAL } \\
\cline { 2 - 7 } & $\mathbf{N}^{\mathbf{o}}$ & $\mathbf{\%}$ & $\mathbf{N}^{\mathbf{0}}$ & $\mathbf{\%}$ & $\mathbf{N}^{\mathbf{0}}$ & $\boldsymbol{\%}$ \\
\hline Sí & 17 & 29,31 & 19 & 43,18 & $\mathbf{3 6}$ & $\mathbf{3 5 , 2 9}$ \\
\hline No & 41 & 70,69 & 25 & 56,82 & $\mathbf{6 6}$ & $\mathbf{6 4 , 7 1}$ \\
\hline TOTAL & $\mathbf{5 8}$ & $\mathbf{1 0 0 , 0 0}$ & $\mathbf{4 4}$ & $\mathbf{1 0 0 , 0 0}$ & $\mathbf{1 0 2}$ & $\mathbf{1 0 0 , 0 0}$ \\
\hline
\end{tabular}

Fuente: Elaboración propia

Continuando con el análisis de estos mercados, pasemos a ver, en la tabla 19 los principales usos que las organizaciones hacen de ellos.

De los datos recogidos en la siguiente tabla 20, destaca la escasa utilización o importancia de los mercados electrónicos dentro del comercio electrónico (solo un 38,89 de las empresas que operan en los mercados electrónicos cierran operaciones comerciales a través de ellos). Ésta está en consonancia con los datos de la economía general, según Ramírez (2012). Según éste autor, la mayoría de las operaciones en el comercio electrónico realizadas por las empresas españolas se llevan a cabo a través de tiendas electrónicas (un 71 por 100 de las compras online y un 73 por 100 de las ventas online). No obstante, tan solo un 25,30 por 100 de las empresas que vende por Internet y un 28 por 100 de las que compra hacen uso de los mercados electrónicos. La gran mayoría, en cambio, prefiere utilizar el comercio electrónico tratando directamente con el proveedor/cliente, tanto para vender online como para comprar, con un 88,60 por 100 y un 93,80 por 100 respectivamente (Ramírez, 2012).

Tabla 20. ¿Qué actividades realiza en los mercados electrónicos? (por razón social)

\begin{tabular}{|l|c|c|c|c|c|c|}
\hline \multirow{2}{*}{$\begin{array}{l}\text { Uso de los mercados } \\
\text { electrónicos }\end{array}$} & \multicolumn{2}{|c|}{ S.C.A } & \multicolumn{2}{c|}{ Otras F.J. } & \multicolumn{2}{c|}{ TOTAL } \\
\cline { 2 - 7 } & $\mathbf{N}^{\mathbf{0}}$ & $\mathbf{\%}$ & $\mathbf{N}^{\mathbf{0}}$ & $\mathbf{\%}$ & $\mathbf{N}^{\mathbf{0}}$ & $\mathbf{\%}$ \\
\hline Como canal de información & 11 & 64,71 & 16 & 84,21 & $\mathbf{2 7}$ & $\mathbf{7 5 , 0 0}$ \\
\hline $\begin{array}{l}\text { Promocionar el producto y } \\
\text { contactar con posibles clientes }\end{array}$ & 8 & 47,06 & 8 & 42,11 & $\mathbf{1 6}$ & $\mathbf{4 4 , 4 4}$ \\
\hline Contacto operaciones de venta & 8 & 47,06 & 6 & 31,58 & $\mathbf{1 4}$ & $\mathbf{3 8 , 8 9}$ \\
\hline
\end{tabular}

Fuente: Elaboración propia 


\section{CONCLUSIONES}

Los resultados obtenidos muestran un uso aún limitado por parte de las empresas analizadas de los sistemas electrónicos y telemáticos como herramientas para la gestión comercial, circunstancia que responde, según las compañías estudiadas, a la incompatibilidad del medio con el tipo de producto que ofertan y a la ausencia de una necesidad que justifique el hacer uso de ellas.

Por otra parte, cabe destacar que no existen grandes diferencias en la forma e intensidad de uso de las TIC entre las sociedades cooperativas y el resto de sociedades, aunque existen algunos aspectos que denotan una mayor confianza en el uso de estas tecnologías por parte de las empresas con una forma jurídica distinta a la de sociedad cooperativa.

Esta situación se extiende también en los mercados electrónicos, que muestran cómo las organizaciones de carácter cooperativo son, con respecto al total de las empresas encuestadas, las que menos aprovechan estos espacios. En general, los mercados electrónicos son poco conocidos por gran parte de las empresas estudiadas. Aquéllas que los conocen y hacen uso de ellos son con fines principalmente informativos y de promoción. En este sentido, destaca que las empresas utilicen esta modalidad de mercado para tales fines, en lugar de para operaciones comerciales más cercanas a la transacción.

Respecto a las redes sociales, hay que subrayar que cerca de la mitad de las empresas analizadas las utiliza de forma frecuente, con una finalidad empresarial, a pesar del corto periodo de tiempo que ha transcurrido desde la aparición de estas herramientas, tal y como las conocemos hoy en día. No obstante, el número de empresas con presencia en redes sociales es todavía reducido, si tenemos en cuenta la repercusión que ha alcanzado este tipo de plataformas entre la sociedad y el uso que se hace de ellas en otros sectores. De igual forma que aparece en el resto de servicios electrónicos estudiados, las sociedades cooperativas son las que menos utilizan las redes sociales, circunstancia que resulta paradójica, dado que es este tipo de organización en la que más consenso hay a la hora de considerar que el uso de estas herramientas sociales genera valor para la actividad empresarial.

Como resumen, señalamos que el tejido empresarial oleícola andaluz necesita involucrarse aún más en los procesos comerciales y de comunicación que tienen lugar a través 
de la Web, al objeto de estar en sintonía con el contexto actual caracterizado por el avance tecnológico y la globalización, especialmente por parte del sector cooperativo, que se encuentra en una posición de desventaja en comparación con la situación que acontece en otras formas jurídicas, en lo que respecta al uso y a la utilización de las nuevas tecnologías con fines empresariales, tal y como se desprende de los resultados de este trabajo.

\section{BIBLIOGRAFÍA}

APIGIAN, C.H., RAGU-NATHAN, B.S., RAGU-NATHAN, T.S. y KUNNATHUR, A. (2005) Internet technology: the strategic imperative. Journal of Electronic Commerce Research, Vol. 6, № 2, pp. 123-146.

BRUQUE, S., VARGAS, A., y HERNÁNDEZ, M. J. (2010) La web como herramienta de negocio. Aplicación al sector oleícola español. DYO (Dirección y Organización), № 26, pp. 169-183.

CABAL, C.C. (2013) Análisis y modelización de la adopción de los sistemas de recomendación en el comercio electrónico. Tesis doctoral, Universidad de Granada.

CARR, N.G. (2004) Does IT Matter? Boston: Harvard Business School Press.

CASARES, J. (1998) Comercio electrónico: una visión distinta aunque no muy distante. Distribución y Consumo, № 41, pp. 5-8.

CELAYA, J. (2008) La Empresa en la Web 2.0: el impacto de las redes sociales y las nuevas formas de comunicación online en la estrategia empresarial. Barcelona: Gestión 2000.

CHENG, Y. y XIE, J. (2008) Online consumer review: Word-of-mouth as a new element of marketing communication mix. Management Science, $\mathrm{N}^{\circ}$ 54, pp. 477-791.

CHUI, M., MANYIKA, J., BUGHIN, J., DOBBS, R., ROXBURGH, C., SARRAZIN, H., SANDS, G. y WESTERGREN, M. (2012) The Social Economy: Unlocking Value and Productivity through Social Technologies, McKinsey Global Institute.

CINK SHAKING BUSINESS (2013) Segundo Observatorio sobre el Uso de las Redes Sociales en las Pymes españolas. Madrid: Fundación Banesto.

ECIRCLE Y MEDIACOM SCIENCE (2011) Estudio Europeo De Redes Sociales y E-mail: El diálogo digital con Facebook, Twitter, E-mail y demás redes en 6 países europeos. Parte III. Qué piensan las empresas. Madrid. Disponible en: http://www.tecon.es/archivos/libro-estudio-redes-sociales. Revisado en julio de 2014.

ESTEBAN, C y QUIRÓS, F. (2014) Mapas de las redes sociales y de mensajería instantánea, versión 4, En: iRedes, IV Congreso Iberoamericano sobre Redes Sociales. Burgos. 
FERNÁNDEZ, S. (2011) Dos grados: Networking 3.0. Madrid: LID Editorial empresarial.

FUNDETEC (2012) Análisis sectorial de la implantación de las TIC en la pyme española. Madrid: Ministerio de Industria, Energía y Turismo.

GALLART, V. (2010) Empresa y redes sociales. Comité econòmic i social de la Comunitat Valenciana, $\mathrm{N}^{\mathrm{o}} 58$, pp. 7-11.

GÓMEZ, J. M. y ALEIXANDRE, G. (2014) Economía social y comportamiento innovador: estudio empírico de las empresas de economía social en Castilla y Léon. CIRIEC-España, Revista de Economía Pública, Social y Cooperativa, No 81, pp. 191-216.

GUNELIUS, S. (2011) 30 minutes social media marketing: step by step techniques to spread the words about your business. Estados Unidos: McGraw-Hill.

INTECO (Instituto Nacional de Tecnologías de la Comunicación) (2010) Guía sobre seguridad y privacidad en el comercio electrónico. Madrid: Ministerio de Industria, Energía y Turismo.

JIMÉNEZ, J.A., DEL ÁGUILA, A.R. y PADILLA, A. (2000) Implicaciones estratégicas del comercio electrónico basado en Internet: modelos de negocio y nuevos intermediarios. Información Comercial Española (ICE): Tribuna de Economía, № 783, pp. 63-78.

LAI, I.K.W., TONG, V. y LAI, D. (2011) Trust factors influencing the adoption of Internetbased interorganizational systems. Electronic Commerce Research and Applications, Vol. $10, \mathrm{~N}^{\mathrm{o}} 1$, pp. 85-93.

LIBEROS, E., SOMALO, I., GIL, J., GIL, J., GARCÍA, R. y MERINO, J.A. (2011) El Libro del Comercio Electrónico. Madrid: ESIC.

LOPEZ, A. (2004) Los mercados electrónicos: un nuevo canal para la internacionalización de la empresa. ICEX (Instituto Español de Comercio Exterior), № 813, pp. 115-139.

MAGRAMA. MINISTERIO DE AGRICULTURA, ALIMENTACIÓN Y MEDIO AMBIENTE (2013) Líneas Estratégicas para la Internacionalización del Sector Agroalimentario. $\quad$ http://www.magrama.gob.es/es/ministerio/planes-estrategias/lineasestrategicas-para-la-internacionalizacion-del-sectoragroalimentario/lineas_estrat\%C3\%A9gicas_internacionalizaci\%C3\%B3n_tcm7278627.pdf . Revisado en junio de 2014.

MEDINA, M. J., MOZAS, A., BERNAL, E. y MORAL, E. (2014) Factores determinantes para la exportación en las empresas cooperativas oleícolas andaluzas. CIRIEC-España, Revista de Economía Pública, Social y Cooperativa, № 81, pp. 241-262.

MEHRTENS, J., CRAGG, P.B. y MILLS, A.M. (2001) A model of Internet adoption by SMEs. Information \& Management, № 39, pp. 165-176. 
MILI, S. y RODRÍGUEZ-ZÚÑ̃IGA, M. (2001) Exploring Future Developments in International Olive Oil Trade and Marketing: A Spanish Perspective. An International Journal, Vol. 17, Nº 3, pp. 397-415.

MONTEGUT, Y., CRISTÓBAL, E. y GÓMEZ, M.J. (2013) La implementación de las TIC en la gestión de las cooperativas agroalimentarias: el caso de la provincia de Lleida. REVESCO. Revista de Estudios Cooperativos, № 110, pp. 223-253.

MORAL, E., BERNAL, E. y ALBA, M.V. (2010) Internet y el acceso de las empresas al mercado mundial. International Conference, XII reunión de economía mundial, caminos para superar la crisis global. Lugo.

MOZAS, A. (2004) Introducción al monográfico: economía social y nuevas tecnologías. CIRIEC-España, Revista de Economía Pública, social y Cooperativa, № 49, pp. 6-11.

MOZAS, A.; BERNAL, E. y MURGADO, E.M. (2007) Caracterización de las empresas oleícolas jiennenses con la actividad comercial ON-LINE. I Congreso de la Cultura del Olivo. Instituto de Estudios Giennenses, pp. 457-468.

MOZAS, A. y BERNAL, E. (2008) El e-business en el cooperativismo agrario: el caso del sector oleícola. Estudios de Economía Aplicada, № 26, pp. 56-68.

NETI, S. (2011) Social media and its role in marketing. International Journal of Enterprise Computing and Business Systems, Vol. 1, № 2, pp. 1-15.

O'REILLY, T. (2005) What Is Web 2.0? Design Patterns and Business Models for the Next Generation of Software. http://oreilly.com/web2/archive/what-is-web-20.html. Revisado en junio de 2014.

PONCE, J. y ESCANCIANO, J. (1997) Comercio electrónico: revolución empresarial. Boletín Fundesco, pp. 188-189.

RAMÍREZ, I. (2012) El comercio electrónico en España - 2012. Emarket Services, Instituto Español de Comercio Exterior (ICEX).

http://www.emarketservices.es/icex/cma/contentTypes/common/records/mostrarDocumen to/?doc $=4644892$.

RASHEED, H.S. (2005) Foreign entry mode and performance: the moderating effects of environment. Journal of Small Business Management, Vol. 43, № 1, pp. 41-51.

SADOWSKI, B.M., MAITLAND, C. y VAN DONGEN, J. (2002) Strategic use of the Internet by small and medium sized companies: an exploratory study. Information Economics and Policy, $\mathrm{N}^{\circ} 14$, pp. 75-93.

SANDULLI, F.D. (2011) Los modelos de negocio de los mercados electrónicos en el sector de la alimentación en España. Distribución y Consumo, Vol. 21, № 116, pp. 33-42. 
SELLERS, R. y AZORÍN, A. (2001) El comercio electrónico y el futuro del canal de distribución turístico. Investigaciones Europeas de Dirección y Economía de la Empresa, Vol. 7, No 1, pp. 13-36.

STANSFIELD, M. y GRANT, K. (2003) An investigation into issues influencing the use of the Internet and electronic commerce among small-medium sized enterprises. Journal of Electronic Commerce Research, Vol. 4, № 1, pp. 15-33.

TRABOLD, H. (2002) Export intermediation: an empirical test of Peng and Ilinitch. Journal of International Business Studies, Vol. 33, № 2, pp. 327-344.

TURBAN, E., LEE, J.K., KING, D., LIANG, T.P. y TURBAN, D. (2009) Electronic commerce 2010. New Jersey: Prentice Hall Press.

URUEÑA, A. (Coord.) (2013) La Sociedad en Red. Informe Anual 2012. Edición 2013. Observatorio Nacional de las Telecomunicaciones y de la Sociedad de la Información (ONTSI). Madrid: Ministerio de Industria, Energía y Turismo.

VÁZQUEZ, T. (2010) Servicios de internet para las empresas oleícolas. La tienda virtual. Revista de Estudios Empresariales. Segunda Época, No 1, pp. 169-188.

WEI, C., ZHANG, C. y SUTANTO, J. (2013) The influence of user interaction and participation in social on the consumption intention of niche products. Information \& Management, $\mathrm{N}^{\circ} .50$, pp. 661-672. 